\title{
A Network Pharmacology-Based Strategy for Predicting Active Ingredients and Potential Targets of Shuilu Erxian Dan in Treating Diabetic Kidney Disease
}

\section{Tingchao Wu}

Hospital of Chengdu University of Traditional Chinese Medicine

Rensong Yue ( $\boldsymbol{\nabla}$ songrenyue@cdutcm.edu.cn)

Hospital of Chengdu University of Traditional Chinese Medicine https://orcid.org/0000-0001-96631859

Mingmin He

Hospital of Chengdu University of Traditional Chinese Medicine

\section{Research}

Keywords: Shuilu Erxian Dan; diabetic kidney disease; diabetic nephropathy; network pharmacology; molecular docking

Posted Date: August 7th, 2020

DOI: https://doi.org/10.21203/rs.3.rs-54579/v1

License: (c) (i) This work is licensed under a Creative Commons Attribution 4.0 International License.

Read Full License 


\section{Abstract \\ Background and objective:}

Recent years, some Chinese scholars have applied Shuilu Erxian Dan (SED) to the treatment of treating diabetic kidney disease (DKD) and achieved well curative effect. However, these studies are mostly limited to clinical observation. This study aimed to explore the molecular mechanisms of SED in treating DKD.

\section{Methods}

The active components of SED were retrieved in TCMSP database and BATMAN-TCM database, and the herbal targets were obtained by drugbank database and SwissTargetPrediction platform. The gene expression data of DKD patients were downloaded from GEO database and analyzed to obtain DKDrelated targets. The ingredient-target network and the PPI network were constructed by Cytoscape software. The clusterProfiler package of $\mathrm{R}$ software is used for bioinformatic analysis. Molecular docking was further applied to verify the interaction between compounds and targets by Autodock Vina software.

\section{Results}

610 differential expressed genes of DKD patients were obtained, and 29 potential targets of SED against DKD were screened out (including PPTGS2, FABP3, HSD17B2, FABP1, HSD11B2, CYP27B1, JUN, UGT2B7, VCAM1, CA2, MAOA, MMP2, CXCR1, SLC22A6, EPHX2, SLC47A1, FOS, EGF, CCL2, COL3A1, GSTA1, GSTA2, HSPA1A, DAO, ALDH2, ALB, GPR18, FPR2, and LPL). All the active ingredients in SED can act on the DKD-related targets, among which quercetin, Ellagic acid, and kaempferol may be the key active compounds. SED may play a therapeutic role in DKD by regulating pathways including "Fluid shear stress and atherosclerosis", "AGE - RAGE signaling pathway in diabetic complications" and "IL-17 signaling pathway".

\section{Conclusion}

This study suggests that the mechanism of SED treating DKD is a complex network with multi-target and multi-pathway, which provides a reference for future experimental studies.

\section{Introduction}

According to the IDF Diabetes Atlas (9th edition), approximately 463.0 million adults aged $20-79$ years worldwide ( $9.3 \%$ of all adults in this age group) have diabetes mellitus (DM), and it is estimated that this number will increase to 700.2 million (10.9\%) in 2045 [1]. Diabetic kidney disease (DKD) refers to chronic kidney disease caused by diabetes [2], and is one of the most common diabetic microvascular 
complications. DKD was formerly known as diabetic nephropathy (DN). In 2014, the American diabetes Association (ADA) and the National Kidney Foundation (KNF) reached a consensus and officially renamed DKD [2]. The data [3] revealed that DKD was found in $20 \%-40 \%$ of DM patients. DKD is characterized by increased glomerular filtration rate and urinary albumin excretion [4], and the lesions can affect the whole kidney (including glomerulus, renal tubules, renal vessels and renal interstitium) [5]. Presently, DKD has become the main cause of end-stage renal disease (ESRD) [6, 7], and about $50 \%$ of DKD patients will eventually develop ESRD [8]. The medical cost of ESRD patients is staggering $[9,10]$. Moreover, compared with DM patients without DKD, the cardiovascular risk and mortality of DKD patients were significantly increased [11, 12]. The pathogenesis of DKD is complex and is currently thought to be related to insulin resistance, oxidative stress, chronic inflammation, overactive renin-angiotensinaldosterone system (RAAS), and renal hemodynamics changes $[13,14]$. Currently, the treatment for DKD include reducing cardiovascular risk, controlling blood glucose, controlling blood pressure, and inhibiting the renin-angiotensin system, but their effects are limited $[6,7,15]$.

In China, Chinese herbal medicine is a way for physicians to against DKD, and there is growing evidence to prove that Chinese herbal medicine has its unique advantages in treating DKD [16-19]. Shuilu Erxian Dan (SED) is a traditional Chinese medicine compound, which is composed of two herbs, Fructus Rosae Laevigatae and Semen Euryales. It was first mentioned in "hongshi jiyanfang", an ancient Chinese book of Southern Song Dynasty (AD 1170) [20]. "Shuilu" in Chinese means water and land, respectively referring to the growing environment of the two herbs. Fructus Rosae Laevigatae is the dry ripe fruit of Rosa Laevigata Michx (family Rosaceae), which grows in mountain, while Semen Euryales is the dry ripe seed of Euryale ferox Salisb (family Water-lily), which grows in water. According to the theory of Traditional Chinese Medicine (TCM), SED has the effect of tonifying kidney and astringing, and it was first used to treat spermatorrhea, enuresis and leukorrhagia. Recent years, some Chinese scholars have applied it to the treatment of DKD and achieved well curative effect [21-25]. However, these studies are mostly limited to clinical observation. It is necessary to explore the underlying molecular mechanisms of SED in the treatment of DKD.

Due to the numerous chemical ingredients, Chinese herbal compound often acts on multiple targets of the diseases with complex pathophysiology. Also, it leaves a tremendous challenge for researchers who want to deeply explore the mechanisms behind the efficacy of Chinese herbal compounds. As a new developing subject, network pharmacology was first proposed by Andrew I Hopkins in 2007 [26], which can reveals the complex network connections among drugs, targets and diseases by the techniques of high-throughput screening, omics, network visualization, molecular docking and so on [27]. The systematic and comprehensive characteristics of network pharmacology have something in common with the wholism of TCM. Therefore, in recent years, network pharmacology has gradually penetrated into the research field of Chinese herbal compound $[28,29]$. Our study is the first to identify the bioactive ingredients of SED and explore its mechanisms in DKD treatment by using network pharmacology approach. 


\section{Materials And Methods}

\subsection{Active ingredients screening}

The ingredients of Fructus Rosae Laevigatae and Semen Euryales were searched and collected from "Traditional Chinese Medicine Systems Pharmacology Database and Analysis Platform" [30] (TCMSP, http://tcmspw.com/tcmsp.php, updated on May 31, 2014) and "Bioinformatics Analysis Tool for Molecular mechANism of Traditional Chinese Medicine" [31] (BATMAN-TCM, http://bionet.ncpsb.org/batman-tcm/, updated on January 2016). To screen the active ingredients of SED by the key criteria of oral bioavailability $(\mathrm{OB}) \geq 30 \%$ [32] and drug likeness $(\mathrm{DL}) \geq 0.18$ [33].

\subsection{Identification of herbal targets}

Potential targets of the active ingredients were searched in DrugBank database [34] (https://www.drugbank.ca/, updated on January 03, 2020). Download the 3D structures of the active compounds in PubChem database [35] (https://pubchem.ncbi.nlm.nih.gov), and upload the 3D structures to SwissTargetPrediction online platform [36] (http://www.swisstargetpre- diction.ch) for obtaining the predicted targets of each compound. The targets obtained from the two platforms were summarized and the duplicates were removed, and the target names were uniformly converted to gene symbol through the Uniprot database [37] (https://www.uniprot.org/).

\subsection{Acquisition of DKD-related targets}

The gene expression data (microdissected glomeruli samples) of DKD patients and non-DKD controls were downloaded from GEO database [38] (https://www.ncbi.nlm.nih.gov/gd- s/?term, Series: GSE96804; Platforms: GPL17586; Samples: GSM2544275- GSM2544335). The limma package [39] (Version 3.42.0) of R software (Version 3.6.1) was used for differential expressed analysis of the gene expression profile. Genes with FDR $<0.05$ and |log 2(fold change) | $>1$ were considered as DKD-related targets.

\subsection{Construction of ingredient-target network and PPI network}

The compound targets were intersected with the DKD-related targets to obtain the SED-DKD intersection targets. Then, the data of the compounds and its corresponding intersection targets was imported into the Cytoscape 3.7.2 software [40] to construct the ingredient-target network, and the network topology parameters were calculated by the "NetworkAnalyzer" tool [41]. The PPI network of SED-DKD intersection targets was constructed by BisoGenet 3.0.0 plugin [42] of Cytoscape software, which was set as "input nodes and its neighbors". PPI data sources include "Biological General Repository for Interaction Datasets" [43] (BioGRID), "IntAct Molecular Interaction Database" [44] (IntAct), "Database of Interacting Proteins" [45] (DIP), "Human Protein Reference Database” [46] (HPRD), "Biomolecular Interaction Network Database" [47] (BIND) and "The Molecular INTeraction Database" [48] (MINT). The parallel edges and selfloops in the PPI network were then removed and the topology parameters were calculated using the 
CytoNCA plugin [49]. Degree centrality (DC) and betweenness centrality (BC) were used as screening indicators to obtain the final core PPI network.

\subsection{Bioinformatic analysis}

First, gene symbols were converted to Entrez IDs using the "org.Hs.eg.db" package (Version 3.8.2) of R software, and then the "clusterProfiler" package [50] (Version 3.12.0) was used for GO and KEGG pathway enrichment analysis of the SED-DKD intersection targets with FDR $<0.05$. The pathway-target network were visualized by Cytoscape software.

\subsection{Molecular docking}

The SED-DKD intersection targets in the core PPI network and the targets with the highest degree in the pathway-target network were used as receptors for molecular docking with the corresponding compounds. The 3D structures of the compounds were constructed by ChemBio3D Ultra 14.0 software and optimized with MMFF94 force field. The 3D structures of the target proteins were downloaded from RCSB Protein Data Bank [51] (http://www.rcsb.org). And AutoDockTools 1.5.6 software was used to pretreat the proteins, including removal water molecules, removal ligand molecules, and protonate 3D hydrogenation. The semi-flexible docking calculation was carried out using Autodock Vina 1.1.2 [52], the exhaustiveness set to 20 , and the rest use default parameters. The conformations with the lowest binding energy were analyzed and plotted by Molecular Operating Environment (MOE) 2015 [53].

\section{Result}

\subsection{Active ingredients and targets of SED}

8 active ingredients of Fructus Rosae Laevigatae and 2 active ingredients of Semen Euryales were retrieved. The Mol ID, molecule name, OB value, DL value, ingredient source and database source of each ingredient are shown in Table 1. And a total of 527 targets of Fructus Rosae Laevigatae and 184 targets of Semen Euryales were obtained.

Table 1. Active ingredients in SED 


\begin{tabular}{|c|c|c|c|c|c|}
\hline Mol ID & Ingredient & $\begin{array}{l}\text { OB } \\
(\%)\end{array}$ & $\mathrm{DL}$ & Herbal & Database \\
\hline MOL008622 & Methyl trametenolate & 42.88 & 0.82 & $\begin{array}{c}\text { Fructus Rosae } \\
\text { Laevigatae }\end{array}$ & $\begin{array}{c}\text { TCMSP, } \\
\text { BATMAN-TCM }\end{array}$ \\
\hline MOL000358 & beta-sitosterol & 36.91 & 0.75 & $\begin{array}{c}\text { Fructus Rosae } \\
\text { Laevigatae }\end{array}$ & TCMSP \\
\hline MOL000098 & quercetin & 46.43 & 0.28 & $\begin{array}{c}\text { Fructus Rosae } \\
\text { Laevigatae }\end{array}$ & TCMSP \\
\hline MOL008628 & $\begin{array}{c}\text { 4'-Methyl-N- } \\
\text { methylcoclaurine }\end{array}$ & 53.43 & 0.26 & $\begin{array}{c}\text { Fructus Rosae } \\
\text { Laevigatae }\end{array}$ & $\begin{array}{c}\text { TCMSP, } \\
\text { BATMAN-TCM }\end{array}$ \\
\hline MOL000422 & kaempferol & 41.88 & 0.24 & $\begin{array}{c}\text { Fructus Rosae } \\
\text { Laevigatae }\end{array}$ & TCMSP \\
\hline MOL005030 & gondoic acid & 30.7 & 0.2 & $\begin{array}{c}\text { Fructus Rosae } \\
\text { Laevigatae }\end{array}$ & TCMSP \\
\hline MOL001494 & Mandenol & 42 & 0.19 & $\begin{array}{c}\text { Fructus Rosae } \\
\text { Laevigatae }\end{array}$ & TCMSP \\
\hline MOL001002 & Ellagic acid & 43.06 & 0.43 & $\begin{array}{c}\text { Fructus Rosae } \\
\text { Laevigatae }\end{array}$ & BATMAN-TCM \\
\hline MOL002773 & beta-carotene & 37.18 & 0.58 & Semen Euryales & TCMSP \\
\hline MOL007180 & vitamin-e & 32.29 & 0.7 & Semen Euryales & TCMSP \\
\hline
\end{tabular}

\subsection{DKD-related targets}

610 DKD-related targets were identified by analyzing the gene expression arrays data downloaded from GEO database. The distribution of the differentially expressed genes is shown in Fig. 1, the red dots in the figure represent the up-regulated genes in DKD patients, the green dots represent the down-regulated genes, and the black dots represent the genes with insignificant differences.

\subsection{Ingredient-target network}

There are twenty-nine intersection genes of SED targets and DKD-related targets, including PPTGS2, FABP3, HSD17B2, FABP1, HSD11B2, CYP27B1, JUN, UGT2B7, VCAM1, CA2, MAOA, MMP2, CXCR1, SLC22A6, EPHX2, SLC47A1, FOS, EGF, CCL2, COL3A1, GSTA1, GSTA2, HSPA1A, DAO, ALDH2, ALB, GPR18, FPR2, and LPL. The ingredient-target network is shown in Fig. 2, the edges between the nodes represent the compound-target interaction. All of the 10 active ingredients we retrieved could act on the corresponding DKD-related targets. Degree is one of the most important topological parameters, the node 
degree of a node is the number of edges linked to it [54]. The three compounds with the highest degree value in the ingredient-target network are quercetin, Ellagic acid and kaempferol, which respectively act on 12,9 , and 8 targets.

\subsection{PPI network}

Figure 3. PPI network. Note: (A) The PPI network of SED-DKD Intersection targets. (B) The PPI network extracted from A with degree centrality $(D C)>48$. (C) The core PPI network extracted from B with betweenness centrality $(B C)>157.9$. The pink node represents that the node belongs to one of the SEDDKD intersection targets.

\subsection{Bioinformatics analysis}

Two hundred and twenty-two GO terms were significantly enriched, 190 in Biological Process (BP), 10 in Cellular Components (CC), and 22 in Molecular Function (MF). The complete data of GO analysis are shown in Supplementary Table 1, and part of the results are shown in Fig. 4. The results of KEGG pathway analysis show that the 20 targets could be mapped to 27 signaling pathways, as shown in Fig. 5. Larger size of the node in Fig. 5 represents the higher degree value.

\subsection{Molecular docking}

As shown in Fig. 3C, the SED-DKD intersection targets in the core PPI network include JUN, FOS, VCAM1 and HSPA1A. The three target proteins with the highest degree value in the pathway-target network (Fig. 6) are JUN, FOS and CCL2, and the degrees are 15, 14 and 7, respectively. FOS, JUN, VCAM1, HSPA1A and CCL2 were used as receptor proteins for molecular docking with the corresponding compounds (Fig. 2). The results of molecular docking are shown in Table 2, and the specific docking modes are shown in Fig. 6.

Table 2. Molecular docking 


\begin{tabular}{|c|c|c|c|c|c|c|}
\hline \multirow[t]{2}{*}{ Compound } & \multirow[t]{2}{*}{ Structure } & \multicolumn{5}{|c|}{ Blinding Energy $\square \mathrm{kcal} \cdot \mathrm{mol}^{-1} \square$} \\
\hline & & FOS & JUN & VCAM1 & HSPA1A & CCL2 \\
\hline beta-sitosterol & & I & -6.1 & I & I & I \\
\hline quercetin & & -5.1 & -6.0 & -5.5 & l & -7.0 \\
\hline 4'-Methyl-N-methylcoclaurine & & l & -5.8 & I & 1 & I \\
\hline kaempferol & & l & -6.2 & -5.4 & I & I \\
\hline Ellagic acid & & I & I & I & -8.7 & I \\
\hline beta-carotene & & l & -7.0 & l & 1 & 1 \\
\hline
\end{tabular}

Note: "/" indicates that the ingredient was not found to act on the protein in step 2.2, so docking is unavailable.

\section{Discussion}

In ancient China, there was no disease name of DKD, which was mostly classified as "edema", "consumptive disease", "Guan Ge" and other categories [56]. According to theory of TCM, the key pathogenesis of DKD lies in the deficiency of kidney qi and the loss of storing function [56-58]. Fructus Rosae Laevigatae can astringe and preserve the kidney essence [59], Semen Euryales can benefit the kidney to preserve the essence [60]. The compatibility of the two herbs provides synergistic effect. The increase of urinary albumin excretion is one of the important diagnostic and evaluation indicators of DKD, strategies that can reduce albuminuria are associated with renal protection [61-66] and additional cardiovascular protection [67] in DKD patients. TCM believes that the kidney as the storehouse of essential qi stores essence and is the root of storage [68]. Albumin is one of the essence of human body. Due to the deficiency of kidney qi and the loss of storing function, the albumin of DKD patients is lost in 
urination. By tonifying kidney and astringing, SED can gradually restore the renal physiological function of storing essence and reduce the albuminuria. Jinsong Jin et al. [69] proved that SED extract could effectively reduce the albuminuria and improve the nutritional status in adriamycin-inducded nephropathy rats. In addition, it was found that Fructus Rosae Laevigatae played a protective role in the kidney of streptozotocin-induced DKD rats by inhibiting oxidative stress [70]. Semen Euryales may reduce albuminuria and delay the progress of DKD by up-regulating the expression of renal SOCS-3 and inhibiting the overexpression of renal IGF-1 in rats [71]. However, due to the "multi-ingredient, multi-target" characteristics of Chinese herbal compound, these studies cannot reveal the mechanisms of SED acting on DKD comprehensively and systematically.

By constructing the ingredient-target network (Fig. 2), we found that all the active compounds in SED can act on the DKD-related targets, which indicated that SED has a strong pertinence in treating DKD. All of the 10 active compounds can affect multiple targets, among which quercetin, Ellagic acid and kaempferol can act on the most targets, so these three compounds may be the crucial active ingredients of SED in treating DKD. In addition, many of these compounds have common targets, suggesting that different compounds may provide synergistic effects. Quercetin belongs to the flavonol group of polyphenolic compounds, which functions as antibacterial, antiviral, anti-inflammatory, antioxidant, anticancer, anti-diabetic, immunomodulatory, etc [72]. The existing studies [73-75] have provided convincing evidence on the renoprotective effects of quercetin in both animal and cell models of DKD. In addition to its antiviral, anti-inflammatory, antioxidant, anti-cancer and anti-diabetic properties, Ellagic acid also exerts hepatoprotective effect [76]. Ellagic acid has been shown to ameliorate renal function and renal pathology in streptozotocin-induced DKD rats by inhibiting the NF-KB pathway and the accumulation of AGEs (advanced glycation end products) in kidney [77-79]. Kaempferol has similar pharmacological effects to quercetin and is used in the treatment of diabetes, metabolic syndrome, liver injury, cancer, etc [80]. Sharma $D$ et al. [81] found that Kaempferol can attenuate DKD by inhibiting RhoA/Rho-kinase mediated inflammatory signaling in vitro.

In this study, we identified 29 potential targets of SED acting on DKD, and constructed the PPI networks of these 29 target proteins and their related proteins. The result shows that the target proteins can interact with each other, and there are as many as 1,399 proteins related to them, which form a complex interaction network. By calculating topological parameters of the network and screening the proteins, we obtained a core PPI network containing 209 proteins (Fig. 4C). Four SED-DKD intersection targets are included in the core PPI network: JUN, FOS, VCAM1 and HSPA1A. Activator protein-1 (AP-1) belongs to the basic-leucine-zipper family of transcription factors, and the most common form of AP-1 is a dimer of JUN protein and FOS protein [82]. High glucose/Ang®-mediated activation of AP-1 can lead to the proliferation of mesangial cells and the excessive accumulation of extracellular matrixs, which is a key pathologic feature of DKD $[83,84]$. The full name of VCAM1 is vascular cell adhesion molecule 1 . During the activation or damage of vascular endothelial cells, VCAM1 can be shed from the cell surface into the circulation, and soluble VCAM1 in the blood of DKD patients was found to be significantly increased, which was positively correlated with UACR (urine albumin: creatinine ratio) $[85,86]$. HSPA1A is an 
inflammation related protein, which is incriminated in the renal inflammation of DKD as the endogenous TLR ligand [87, 88].

The GO analysis of SED-DKD intersection targets enriched some interesting GO terms, such as "fatty acid metabolic process", "regulation of lipid metabolic process", "peroxisome", "SMAD binding" and "glutathione transferase activity". In nonadipose tissues, excess cytosolic free fatty acids (FFAs) can lead to cell dysfunction and death, a process known as "lipotoxicity". Disturbed FFA metabolism and renal lipid accumulation are thought to be associated with DKD glomerulosclerosis and tubulointerstitial damage in DKD [89-91]. Peroxisome is a kind of microbody whose main function is to catalyze the $\beta$ oxidation of fatty acids and the hydrolysis of hydrogen peroxide [92]. The inactivation of peroxisomal catalase in DKD animal models can cause alterations of mitochondrial membrane potential, which stimulate the generation of mitochondrial reactive oxygen species (ROS) [93]. Oxidative stress caused by excessive ROS production is considered to be an important factor in the occurrence and development of diabetic complications including diabetic nephropathy [94-97]. Members of the SMAD protein family act as signal integrators and interact with several DKD-related signaling pathways, among which Smad3 is pathogenic, Smad2 and Smad7 are protective $[98,99]$. Glutathione-S-transferases represent a superfamily of enzymes involved in cell protection and detoxification, play an important role in protecting the body from oxidative stress products [100]. Glutathione-S- transferase activity is considered as one of the markers of severity in DKD patients [101].

Multiple signaling pathways were significantly enriched by KEGG analysis, among which "Fluid shear stress and atherosclerosis" is an important atherosclerosis-related pathway. DKD is closely related to cardiovascular disease. Microalbuminuria reflects generalized endothelial damage and is regarded as an early event in atherosclerosis [102]. Vascular endothelial dysfunction is also considered to be involved in the pathogenesis of DKD [103]. In addition, several traditional risk factor for atherosclerosis has been identified in DKD patients including high blood pressure, hyperlipaemia and procoagulatory state associated with endothelial dysfunction [104]. A lot of evidence supports the significance of "AGE - RAGE signaling pathway" in the pathogenesis of DKD, and its blockade seems to be an attractive therapeutic target [105]. "IL-17 signaling pathway" is believed to play a pro-inflammatory role in podocyte injury, mesangial expansion and renal fibrosis in DKD patients [106].

In our study, molecular docking was further applied to verify the interaction between compounds and targets. The combination with the lower binding energy scores is more stable, and the binding energy $\leq-$ $5.0 \mathrm{kcal} \cdot \mathrm{mol}^{-1}$ was defined as the standard of well binding between ligands and receptors in some studies [107-109]. As shown in Table 2, the binding energies of all docking are less than $-5 \mathrm{kcal} \cdot \mathrm{mol}^{-1}$. Take the complex with the lowest binding energy as an example, "Ellagic acis-HSPA1A" (Fig. 6I) was stabilized by five $\mathrm{H}$-bonds with residues including Glu 175, Thr 13, Thr 14 and Asp 366.

\section{Conclusion}


This study explored the mechanism of SED in the treatment of DKD by means of network pharmacology. We identified 29 potential targets of SED acting on DKD, among which FOS, JUN, VCAM1, HSPA1A and CCL2 were the key targets. SED may play a therapeutic role in DKD by regulating pathways including "Fluid shear stress and atherosclerosis", "AGE - RAGE signaling pathway in diabetic complications" and "IL-17 signaling pathway". Quercetin, Ellagic acid, and kaempferol in SED may be the key active ingredients. The mechanism of SED treating DKD is a complex network with multi-target and multipathway. This study provides a scientific theoretical basis for the prevention and treatment of SED acting on DKD, and also provides a reference for researchers in related fields to further carry out experimental work.

\section{Abbreviations}

SED

Shuilu Erxian Dan; DKD:diabetic kidney disease; DM:diabetes mellitus; ESRD:end-stage renal disease; TCM:Traditional Chinese Medicine; OB:oral bioavailability; DL:drug likeness; DC:Degree centrality; BC:betweenness centrality; AGEs:advanced glycation end products; AP-1:Activator protein-1; VCAM1:vascular cell adhesion molecule 1; UACR:urine albumin:creatinine ratio ; FFAs:excess cytosolic free fatty acids; ROS:reactive oxygen species.

\section{Declarations}

\section{Ethics approval and consent to participate}

Not applicable.

\section{Consent for publication}

Not applicable.

\section{Availability of data and materials}

All data are available in the manuscript and they are showed in figures, tables and supplement file.

\section{Competing interests}

The authors declare that they have no conflict of interest.

\section{Funding}

This study was supported by the National Natural Science Foundation of China (no. 81774279).

\section{Authors' contributions}


TW and RY: study design; acquisition of data; analysis and interpretation of data; drafting of the manuscript; critical revision of the manuscript for important intellectual content; statistical analysis. $\mathrm{MH}$ : revision of the manuscript and study supervision. The author(s) read and approved the final manuscript.

\section{Acknowledgements}

Not applicable.

\section{References}

1. Saeedi P, Petersohn I, Salpea P, et al. Global and regional diabetes prevalence estimates for 2019 and projections for 2030 and 2045: Results from the International Diabetes Federation Diabetes Atlas, 9(th) edition. Diabetes research and clinical practice. 2019;157:107843.

2. Tuttle KR, Bakris GL, Bilous RW, et al. Diabetic kidney disease: a report from an ADA Consensus Conference. Diabetes care. 2014;37:2864-2883.

3. Gheith O, Farouk N, Nampoory N, Halim MA, Al-Otaibi T. Diabetic kidney disease: world wide difference of prevalence and risk factors. Journal of nephropharmacology. 2016;5:49-56.

4. de Boer IH, Rue TC, Hall YN, Heagerty PJ, Weiss NS, Himmelfarb J. Temporal trends in the prevalence of diabetic kidney disease in the United States. Jama. 2011;305:2532-9.

5. Kanwar YS, Sun L, Xie P, Liu FY, Chen S. A glimpse of various pathogenetic mechanisms of diabetic nephropathy. Annual review of pathology. 2011;6:395-423.

6. Umanath K, Lewis JB. Update on Diabetic Nephropathy: Core Curriculum 2018. American journal of kidney diseases: the official journal of the National Kidney Foundation. 2018;71:884-95.

7. Fu H, Liu S, Bastacky SI, Wang X, Tian XJ, Zhou D. Diabetic kidney diseases revisited: A new perspective for a new era. Molecular metabolism. 2019;30:250-63.

8. Kato M, Natarajan R. Epigenetics and epigenomics in diabetic kidney disease and metabolic memory. Nature reviews Nephrology. 2019;15:327-45.

9. Stanton RC. Clinical challenges in diagnosis and management of diabetic kidney disease. American journal of kidney diseases: the official journal of the National Kidney Foundation. 2014;63:3-21.

10. Schirr-Bonnans S, Costa N, Derumeaux-Burel H, et al. Cost of diabetic eye, renal and foot complications: a methodological review. The European journal of health economics: HEPAC : health economics in prevention care. 2017;18:293-312.

11. Afkarian M, Sachs MC, Kestenbaum B, et al. Kidney disease and increased mortality risk in type 2 diabetes. Journal of the American Society of Nephrology: JASN. 2013;24:302-8.

12. Fox CS, Matsushita K, Woodward M, et al. Associations of kidney disease measures with mortality and end-stage renal disease in individuals with and without diabetes: a meta-analysis. Lancet. 2012;380:1662-73.

13. Lin YC, Chang YH, Yang SY, Wu KD, Chu TS. Update of pathophysiology and management of diabetic kidney disease. Journal of the Formosan Medical Association = Taiwan yi zhi. 2018;117:662-75. 
14. Karalliedde J, Gnudi L. Diabetes mellitus, a complex and heterogeneous disease, and the role of insulin resistance as a determinant of diabetic kidney disease. Nephrology, dialysis, transplantation: official publication of the European Dialysis and Transplant Association -. European Renal Association. 2016;31:206-13.

15. Zagkotsis G, Markou M, Paschou E, Papanikolaou P, Sabanis N. Preventing the development and progression of diabetic kidney disease: Where do we stand? Diabetes metabolic syndrome. 2018;12:585-90.

16. Wang Y, Zhao H, Wang Q, et al. Chinese Herbal Medicine in Ameliorating Diabetic Kidney Disease via Activating Autophagy. J Diabetes Res. 2019;2019:9030893.

17. Lu Z, Zhong Y, Liu W, Xiang L, Deng Y. The Efficacy and Mechanism of Chinese Herbal Medicine on Diabetic Kidney Disease. J Diabetes Res. 2019;2019:2697672.

18. Zhang YL, Shergis J, et al. Chinese herbal medicine for diabetic kidney disease: a systematic review and meta-analysis of randomised placebo-controlled trials. BMJ open. 2019;9:e025653.

19. Wen Y, Yan M, Zhang B, Li P. Chinese medicine for diabetic kidney disease in China. Nephrology (Carlton Vic). 2017;22(Suppl 4):50-5.

20. Hu Y, Wu Q, Yue W, Gu W, Wu C. Experimental Study of Shuilu Erxian Pill in the Treatment of Rats with Deficiency Syndrome. Journal of Nanjing University of Traditional Chinese Medicine. 2014;30:153-5.

21. Chen L. Water and Land Dendroxine Treatment Clinical Observation of Early Diabetic Nephropathy. Medical Innovation of China. 2017;14:111-4.

22. Chen L, Liu C. Clinical Observation on Food Restriction Combined with Liuwei Dihuang Decoction and Shuilu Erxian Dan in Treatment of Diabetic Nephropathy. Journal of Practical Traditional Chinese Internal Medicine. 2019;33:61-3.

23. Zhou B, Ma Y, Song L, Sun X, Zhang L, Xiao H. TCM treatment of diabetic nephropathy. Chinese Journal of Clinical Healthcare. 2010;13:658-60.

24. Cai S. A review of the study on the prevention and treatment of diabetic nephropathy with Shuilu Erxian Dan. Journal of China Prescription Drug. 2019;17:17-8.

25. Huang $X$. Observation on the curative effect of integrated Chinese and western medicine on diabetic nephropathy. Journal of New Chinese Medicine. 2011;43:36-7.

26. Hopkins AL. Network pharmacology. Nature biotechnology. 2007;25:1110-1.

27. Barabasi AL, Gulbahce N, Loscalzo J. Network medicine: a network-based approach to human disease. Nat Rev Genet. 2011;12:56-68.

28. Zhang W, Huai Y, Miao Z, Qian A, Wang Y. Systems Pharmacology for Investigation of the Mechanisms of Action of Traditional Chinese Medicine in Drug Discovery. Front Pharmacol. 2019;10:743.

29. Luo TT, Lu Y, Yan SK, Xiao X, Rong XL, Guo J. Network Pharmacology in Research of Chinese Medicine Formula: Methodology, Application and Prospective. Chin J Integr Med. 2020;26:72-80. 
30. Ru J, Li P, Wang J, et al. TCMSP: a database of systems pharmacology for drug discovery from herbal medicines. J Cheminform. 2014;6:13.

31. Liu Z, Guo F, Wang Y, et al. BATMAN-TCM: a Bioinformatics Analysis Tool for Molecular mechANism of Traditional Chinese Medicine. Scientific reports. 2016;6:21146.

32. Xu X, Zhang W, Huang $C$, et al. A novel chemometric method for the prediction of human oral bioavailability. Int J Mol Sci. 2012;13:6964-82.

33. Tian S, Wang J, Li Y, Li D, Xu L, Hou T. The application of in silico drug-likeness predictions in pharmaceutical research. Adv Drug Deliv Rev. 2015;86:2-10.

34. Wishart DS, Feunang YD, Guo AC, et al. DrugBank 5.0: a major update to the DrugBank database for 2018. Nucleic Acids Res. 2018;46:D1074-82.

35. Kim S, Thiessen PA, Bolton EE, et al. PubChem Substance and Compound databases. Nucleic acids research. 2016;44:D1202-13.

36. Daina A, Michielin O, Zoete V. SwissTargetPrediction: updated data and new features for efficient prediction of protein targets of small molecules. Nucleic acids research. 2019;47:W357-64.

37. The UniProt Consortium. UniProt: the universal protein knowledgebase. Nucleic acids research. 2017;45:D158-69.

38. Barrett T, Wilhite SE, Ledoux P, et al. NCBI GEO: archive for functional genomics data sets-update. Nucleic Acids Res. 2013;41:D991-5.

39. Ritchie ME, Phipson B, Wu D, et al. limma powers differential expression analyses for RNAsequencing and microarray studies. Nucleic Acids Res. 2015;43:e47.

40. Shannon P, Markiel A, Ozier O, et al. Cytoscape: a software environment for integrated models of biomolecular interaction networks. Genome research. 2003;13:2498-504.

41. Assenov Y, Ramirez F, Schelhorn SE, Lengauer T, Albrecht M. Computing topological parameters of biological networks. Bioinformatics. 2008;24:282-4.

42. Martin A, Ochagavia ME, Rabasa LC, Miranda J, Fernandez-de-Cossio J, Bringas R. BisoGenet: a new tool for gene network building, visualization and analysis. BMC Bioinform. 2010;11:91.

43. Oughtred R, Stark C, Breitkreutz BJ, et al. The BioGRID interaction database: 2019 update. Nucleic Acids Res. 2019;47:D529-41.

44. Kerrien S, Aranda B, Breuza L, et al. The IntAct molecular interaction database in 2012. Nucleic Acids Res. 2012;40:D841-6.

45. Salwinski L, Miller CS, Smith AJ, Pettit FK, Bowie JU, Eisenberg D. The Database of Interacting Proteins: 2004 update. Nucleic Acids Res. 2004;32:D449-51.

46. Keshava Prasad TS, Goel R, Kandasamy K, et al. Human Protein Reference Database-2009 update. Nucleic Acids Res. 2009;37:D767-72.

47. Gilbert D. Biomolecular interaction network database. Brief Bioinform. 2005;6:194-8.

48. Licata L, Briganti L, Peluso D, et al. MINT, the molecular interaction database: 2012 update. Nucleic Acids Res. 2012;40:D857-61. 
49. Tang Y, Li M, Wang J, Pan Y, Wu FX. CytoNCA: a cytoscape plugin for centrality analysis and evaluation of protein interaction networks. Bio Syst. 2015;127:67-72.

50. Yu G, Wang LG, Han Y, He QY. clusterProfiler: an R package for comparing biological themes among gene clusters. Omics: a journal of integrative biology. 2012;16:284-7.

51. Burley SK, Berman HM, Bhikadiya C, et al. RCSB Protein Data Bank: biological macromolecular structures enabling research and education in fundamental biology, biomedicine, biotechnology and energy. Nucleic acids research. 2019;47:D464-74.

52. Trott O, Olson AJ. AutoDock Vina: improving the speed and accuracy of docking with a new scoring function, efficient optimization, and multithreading. J Comput Chem. 2010;31:455-61.

53. Vilar S, Cozza G, Moro S. Medicinal chemistry and the molecular operating environment (MOE): application of QSAR and molecular docking to drug discovery. Curr Top Med Chem. 2008;8:1555-72.

54. Doncheva NT, Assenov Y, Domingues FS, Albrecht M. Topological analysis and interactive visualization of biological networks and protein structures. Nature protocols. 2012;7:670-85.

55. Zhang Y, Li Z, Yang M, et al. Identification of GRB2 and GAB1 coexpression as an unfavorable prognostic factor for hepatocellular carcinoma by a combination of expression profile and network analysis. Plos one. 2013;8:e85170-0.

56. Medicine CAoC. The guideline for the prevention and treatment of diabetic nephropathy in Traditional Chinese Medicine. Chinese Medicine Modern Distance Education of China. 2011;9:151-3.

57. Gao X, Shang J, Liu H, Yu B. A Meta-Analysis of the Clinical Efficacy of TCM Decoctions Made from Formulas in the Liuwei Dihuang Wan Categorized Formulas in Treating Diabetic Nephropathy Proteinuria. Evid Based Complement Alternat Med. 2018;2018:2427301.

58. Wang XQ, Zou XR, Zhang YC. From "Kidneys Govern Bones" to Chronic Kidney Disease, Diabetes Mellitus, and Metabolic Bone Disorder: A Crosstalk between Traditional Chinese Medicine and Modern Science. Evid Based Complement Alternat Med. 2016;2016:4370263.

59. Liao A, Zhou G, Chen J, Gao Y, Yu L, Zhang C. Simultaneous Determination of Six Active Compounds in Rosae Laevigatae Fructus by Ultrasonic-Assisted Extraction and HPLC with Gradient Wavelength Detection. Food Science. 2017;38:141-5.

60. Yang X, Zhang Q. Research progress in Euryale ferox: a Chinese herb. Journal of International Pharmaceutical Research. 2015;42:160-4.

61. de Zeeuw D. Albuminuria: a target for treatment of type 2 diabetic nephropathy. Semin Nephrol. 2007;27:172-81.

62. Wada T, Shimizu M, Toyama T, Hara A, Kaneko S, Furuichi K. Clinical impact of albuminuria in diabetic nephropathy. Clin Exp Nephrol. 2012;16:96-101.

63. Campbell RC, Ruggenenti P, Remuzzi G. Proteinuria in diabetic nephropathy: treatment and evolution. Curr Diab Rep. 2003;3:497-504.

64. de Zeeuw D, Remuzzi G, Parving HH, et al. Proteinuria, a target for renoprotection in patients with type 2 diabetic nephropathy: lessons from RENAAL. Kidney international. 2004;65:2309-20. 
65. Eijkelkamp WB, Zhang Z, Remuzzi G, et al. Albuminuria is a target for renoprotective therapy independent from blood pressure in patients with type 2 diabetic nephropathy: post hoc analysis from the Reduction of Endpoints in NIDDM with the Angiotensin II Antagonist Losartan (RENAAL) trial. Journal of the American Society of Nephrology: JASN. 2007;18:1540-6.

66. de Zeeuw D, Ramjit D, Zhang Z, et al. Renal risk and renoprotection among ethnic groups with type 2 diabetic nephropathy: a post hoc analysis of RENAAL. Kidney international. 2006;69:1675-82.

67. de Zeeuw D, Remuzzi G, Parving HH, et al. Albuminuria, a therapeutic target for cardiovascular protection in type 2 diabetic patients with nephropathy. Circulation. 2004;110:921-7.

68. Tan C, Li L, Zhang H. Recognization of the Physiological Nature that Kidney Control Storing From Meridian foudation. Guiding Journal of Traditional Chinese Medicine and Pharmacy. 2008;15-16.

69. Jin J, Sheng L, Jiang N, Wu B, Shen S, Guo X. Experimental Study on Influence of Shuiluerxian Pellet to Adrianmycin-inducded Nephropathy Rat's Proteinuria. Journal of Hubei University of Chinese Medicine. 2013;15:14-7.

70. Zhou Y, Liao Q, Luo Y, Qing Z, Zhang Q, He G. Renal protective effect of Rosa laevigata Michx. by the inhibition of oxidative stress in streptozotocin-induced diabetic rats. Mol Med Rep. 2012;5:1548-54.

71. Han Y, FANG J, SUN Y, Zhang X, Liu W. Effect of Euryale Ferox on the Expression of SOCS-3, IGF-1 in Renal Tissues of Diabetic Nephropathy Rats. Chinese Journal of Integrated Traditional Western Nephrology. 2014;15:767-9.

72. Massi A, Bortolini O, Ragno D, et al. Research Progress in the Modification of Quercetin Leading to Anticancer Agents. Molecules. 2017;22:1270.

73. Jiang X, Yu J, Wang X, Ge J, Li N. Quercetin improves lipid metabolism via SCAP-SREBP2-LDLr signaling pathway in early stage diabetic nephropathy. Diabetes Metab Syndr Obes. 2019;12:82739.

74. Lei $D$, Chengcheng $L$, Xuan Q, et al. Quercetin inhibited mesangial cell proliferation of early diabetic nephropathy through the Hippo pathway. Pharmacol Res. 2019;146:104320-0.

75. Tang L, Li K, Zhang Y, et al. Quercetin liposomes ameliorate streptozotocin-induced diabetic nephropathy in diabetic rats. Scientific reports. 2020;10:2440-0.

76. Derosa G, Maffioli P, Sahebkar A. Ellagic Acid and Its Role in Chronic Diseases. Adv Exp Med Biol. 2016;928:473-9.

77. Ahad A, Ganai AA, Mujeeb M, Siddiqui WA. Ellagic acid, an NF-KB inhibitor, ameliorates renal function in experimental diabetic nephropathy. Chem Biol Interact. 2014;219:64-75.

78. Raghu G, Jakhotia S, Yadagiri Reddy P, Kumar PA, Bhanuprakash Reddy G. Ellagic acid inhibits nonenzymatic glycation and prevents proteinuria in diabetic rats. Food Funct. 2016;7:1574-83.

79. Zhou B, Li Q, Wang J, Chen P, Jiang S. Ellagic acid attenuates streptozocin induced diabetic nephropathy via the regulation of oxidative stress and inflammatory signaling. Food Chem Toxicol. 2019;123:16-27. 
80. Ren J, Lu Y, Qian Y, Chen B, Wu T, Ji G. Recent progress regarding kaempferol for the treatment of various diseases. Experimental therapeutic medicine. 2019;18:2759-76.

81. Sharma D, Gondaliya P, Tiwari V, Kalia K. Kaempferol attenuates diabetic nephropathy by inhibiting RhoA/Rho-kinase mediated inflammatory signalling. Biomed Pharmacother. 2019;109:1610-9.

82. Angel P, Karin M. The role of Jun, Fos and the AP-1 complex in cell-proliferation and transformation. Biochim Biophys Acta. 1991;1072:129-57.

83. Sanchez AP, Sharma K. Transcription factors in the pathogenesis of diabetic nephropathy. Expert Rev Mol Med. 2009;11:e13-3.

84. Ahn JD, Morishita R, Kaneda Y, et al. Transcription factor decoy for AP-1 reduces mesangial cell proliferation and extracellular matrix production in vitro and in vivo. Gene Ther. 2004;11:916-23.

85. Koga M, Otsuki M, Kubo M, Hashimoto J, Kasayama S. Relationship between circulating vascular cell adhesion molecule-1 and microvascular complications in type 2 diabetes mellitus. Diabetic medicine: a journal of the British Diabetic Association. 1998;15:661-7.

86. Wong CK, Ho AW, Tong PC, et al. Aberrant expression of soluble co-stimulatory molecules and adhesion molecules in type 2 diabetic patients with nephropathy. J Clin Immunol. 2008;28:36-43.

87. Jheng H-F, Tsai P-J, Chuang Y-L, et al. Albumin stimulates renal tubular inflammation through an HSP70-TLR4 axis in mice with early diabetic nephropathy. Dis Model Mech. 2015;8:1311-21.

88. Feng Y, Yang S, Ma Y, Bai X-Y, Chen X. Role of Toll-like receptors in diabetic renal lesions in a miniature pig model. Sci Adv. 2015;1:e1400183-3.

89. Sieber J, Jehle AW. Free Fatty acids and their metabolism affect function and survival of podocytes. Front Endocrinol (Lausanne). 2014;5:186-6.

90. Murea M, Freedman BI, Parks JS, Antinozzi PA, Elbein SC, Ma L. Lipotoxicity in diabetic nephropathy: the potential role of fatty acid oxidation. Clin J Am Soc Nephrol. 2010;5:2373-9.

91. Herman-Edelstein M, Scherzer P, Tobar A, Levi M, Gafter U. Altered renal lipid metabolism and renal lipid accumulation in human diabetic nephropathy. Journal of lipid research. 2014;55:561-72.

92. Shamekhi Amiri F. Intracellular organelles in health and kidney disease. Nephrol Ther. 2019;15:9-21.

93. Vasko R. Peroxisomes and Kidney Injury. Antioxid Redox Signal. 2016;25:217-31.

94. Panigrahy SK, Bhatt R, Kumar A. Reactive oxygen species: sources, consequences and targeted therapy in type 2 diabetes. Journal of drug targeting. 2017;25:93-101.

95. Forbes JM, Coughlan MT, Cooper ME. Oxidative stress as a major culprit in kidney disease in diabetes. Diabetes. 2008;57:1446-54.

96. Stanton RC. Oxidative stress and diabetic kidney disease. Curr Diab Rep. 2011;11:330-6.

97. Mima A. Inflammation and oxidative stress in diabetic nephropathy: new insights on its inhibition as new therapeutic targets. J Diabetes Res. 2013;2013:248563.

98. Lan HY. Transforming growth factor-beta/Smad signalling in diabetic nephropathy. Clin Exp Pharmacol Physiol. 2012;39:731-8.

99. Lan HY, Chung ACK. Transforming growth factor-beta and Smads. Contrib Nephrol. 2011;170:75-82. 
100. Sharma M, Gupta S, Singh K, et al. Association of glutathione-S-transferase with patients of type 2 diabetes mellitus with and without nephropathy. Diabetes metabolic syndrome. 2016;10:194-7.

101. Tesauro M, Nisticò $S$, Noce A, et al. The possible role of glutathione-S-transferase activity in diabetic nephropathy. Int J Immunopathol Pharmacol. 2015;28:129-33.

102. Jensen JS. Microalbuminaria and the risk of atherosclerosis. Clinical epidemiological and physiological investigations. Dan Med Bull. 2000;47:63-78.

103. Balakumar P, Chakkarwar VA, Krishan P, Singh M. Vascular endothelial dysfunction: a tug of war in diabetic nephropathy? Biomed Pharmacother. 2009;63:171-9.

104. Czekalski S. Diabetic nephropathy and cardiovascular diseases. Rocz Akad Med Bialymst. 2005;50:122-5.

105. Sanajou D, Ghorbani Haghjo A, Argani H, Aslani S. AGE-RAGE axis blockade in diabetic nephropathy: Current status and future directions. Eur J Pharmacol. 2018;833:158-64.

106. Ma J, Li YJ, Chen X, Kwan T, Chadban SJ, Wu H. Interleukin 17A promotes diabetic kidney injury. Scientific reports. 2019;9:2264-4.

107. Li X, Xu X, Wang J, et al. A system-level investigation into the mechanisms of Chinese Traditional Medicine: Compound Danshen Formula for cardiovascular disease treatment. PLoS One. 2012;7:e43918.

108. Li B, Rui J, Ding X, Yang X. Exploring the multicomponent synergy mechanism of Banxia Xiexin Decoction on irritable bowel syndrome by a systems pharmacology strategy. J Ethnopharmacol. 2019;233:158-68.

109. Xu SN, Zhuang L, Zhai YY, et al. Material Basis and Mechanism of Erzhi Pill for Preventing Osteoporosis Based on Network Pharmacology. Chinese Pharmaceutical Journal. 2019;53:34-41.

\section{Figures}




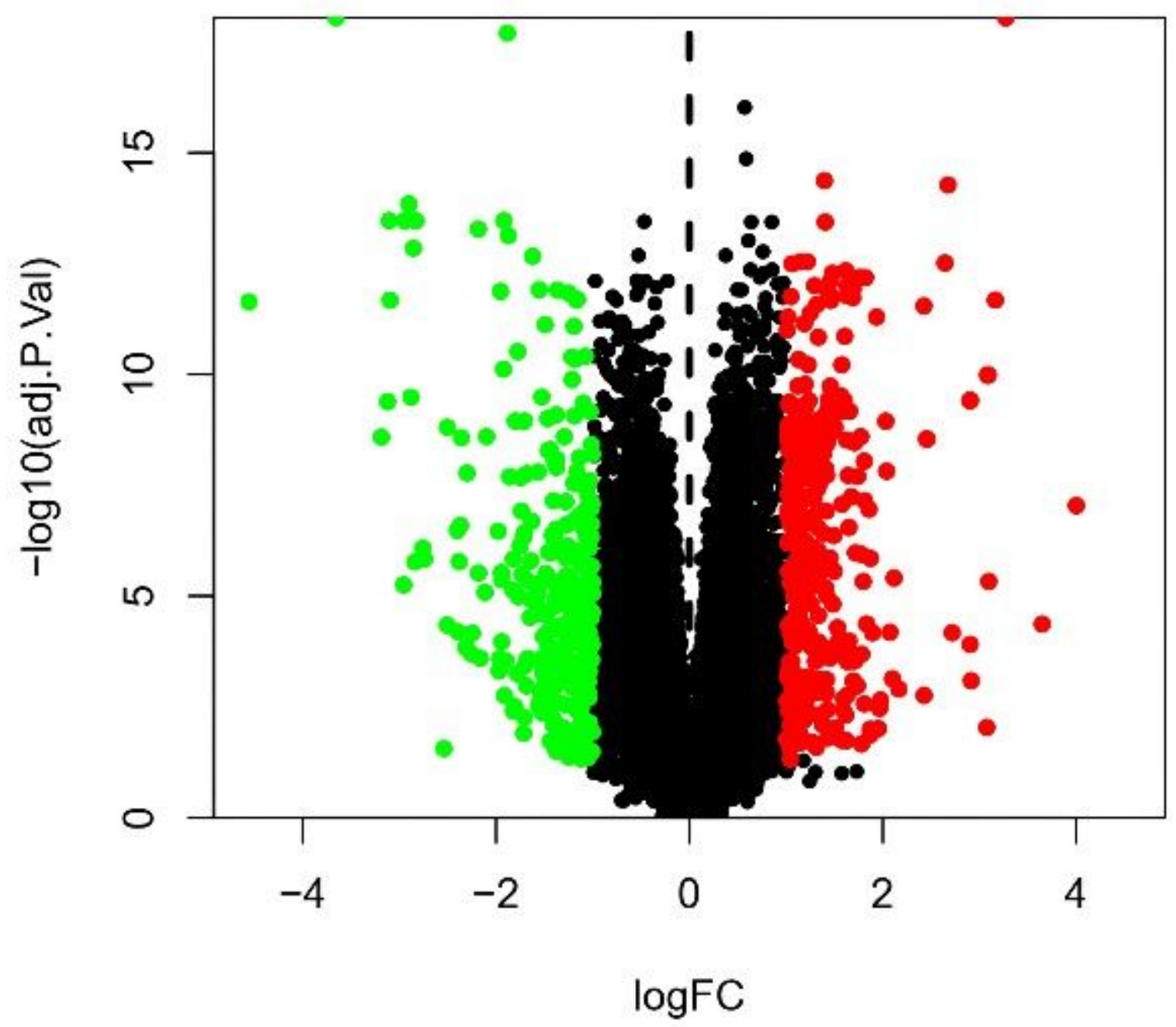

Figure 1

Volcano plot of DKD-Related differentially expressed genes. Note: The abscissa represents the fold changes in gene expression and the ordinate represents the statistical significance of the variations in gene expression. 


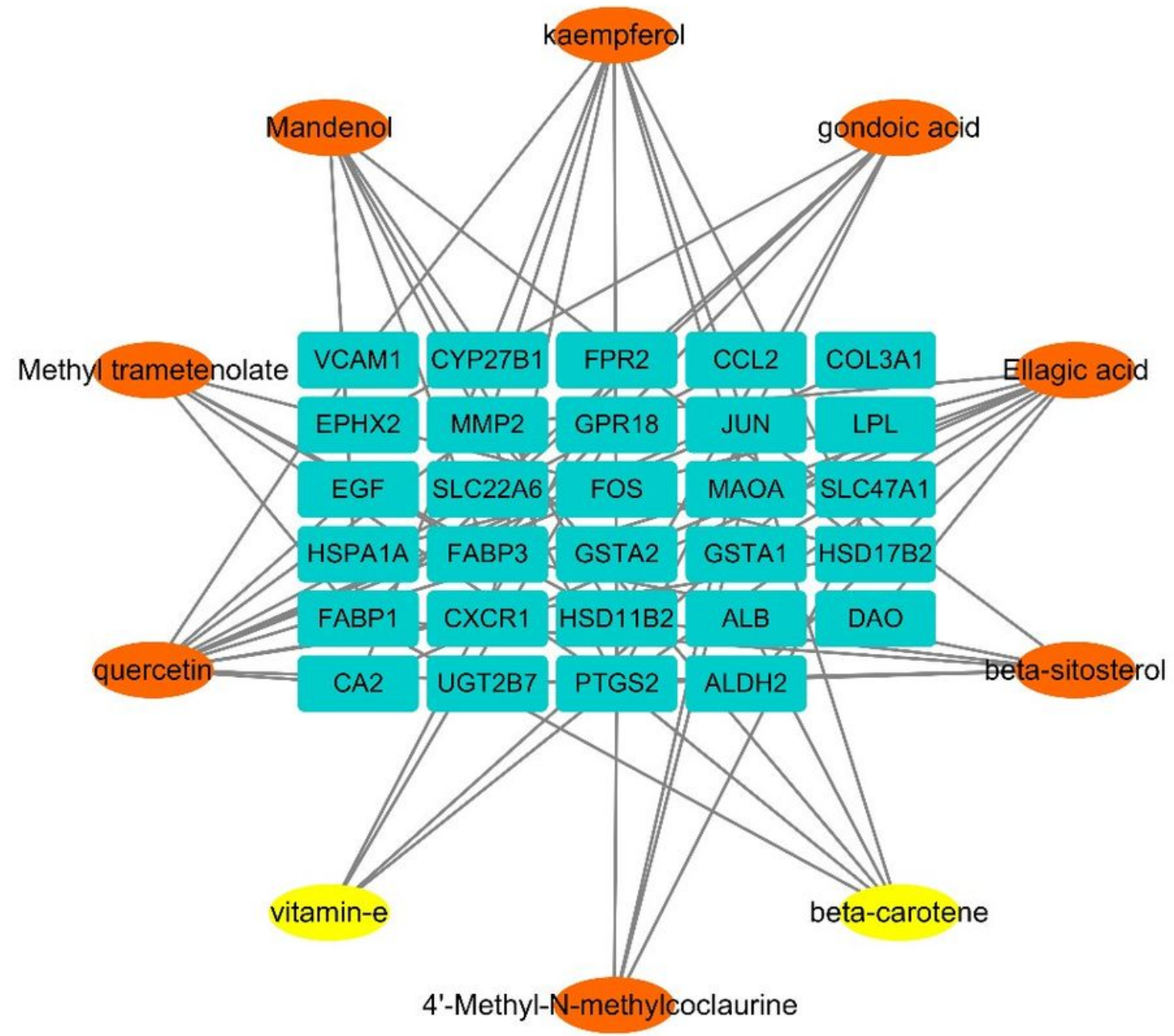

Figure 2

Ingredient-target network. Note: The blue-green rectangles represent intersection targets, the orange ovals represent compounds from Fructus Rosae Laevigatae, the yellow ovals represent compounds from Semen Euryales. 

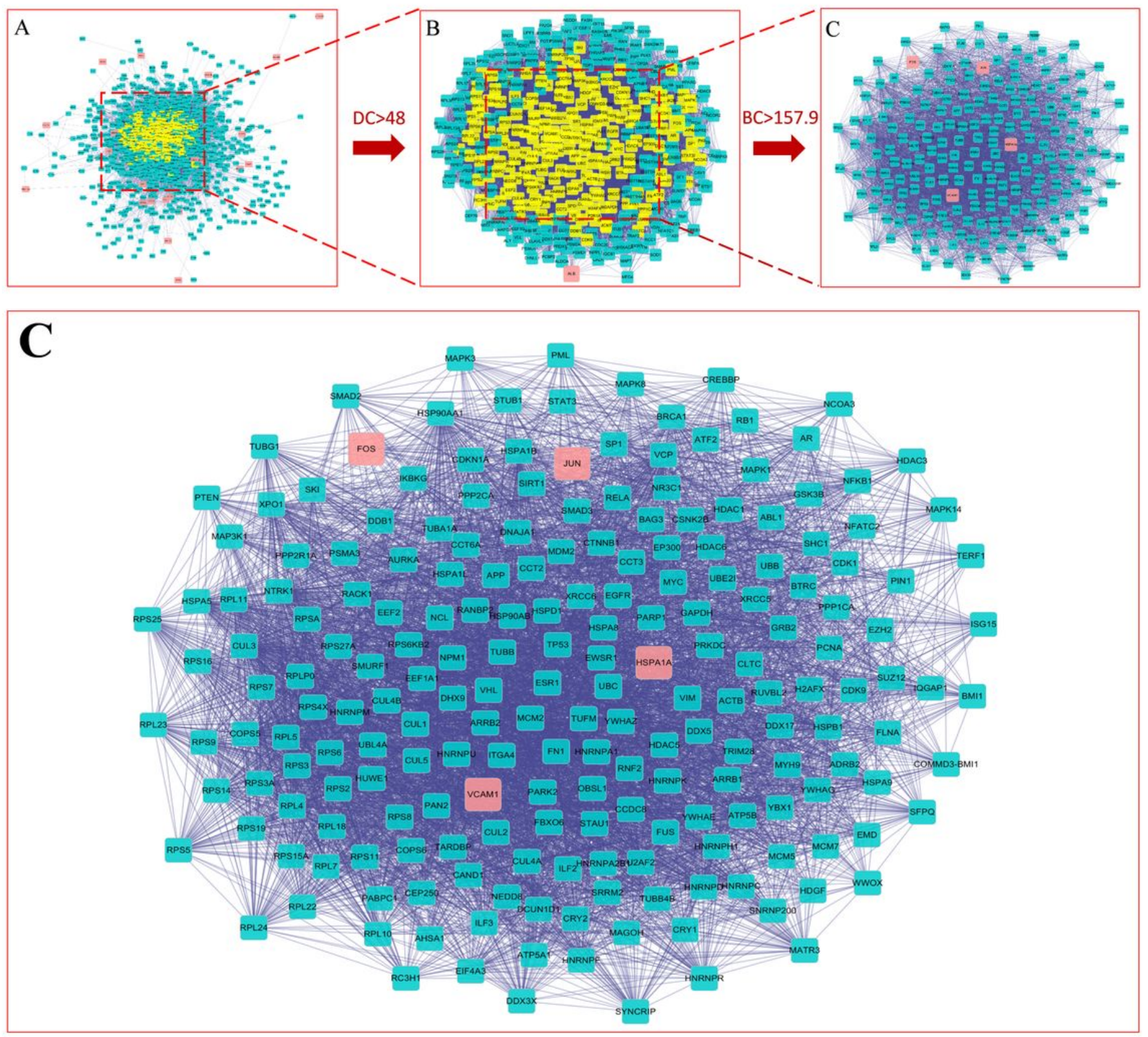

\section{Figure 3}

PPI network. Note: (A) The PPI network of SED-DKD Intersection targets. (B) The PPI network extracted from A with degree centrality (DC) $>48$. (C) The core PPI network extracted from $B$ with betweenness centrality $(B C)>157.9$. The pink node represents that the node belongs to one of the SED-DKD intersection targets. 
(A) Biological Process

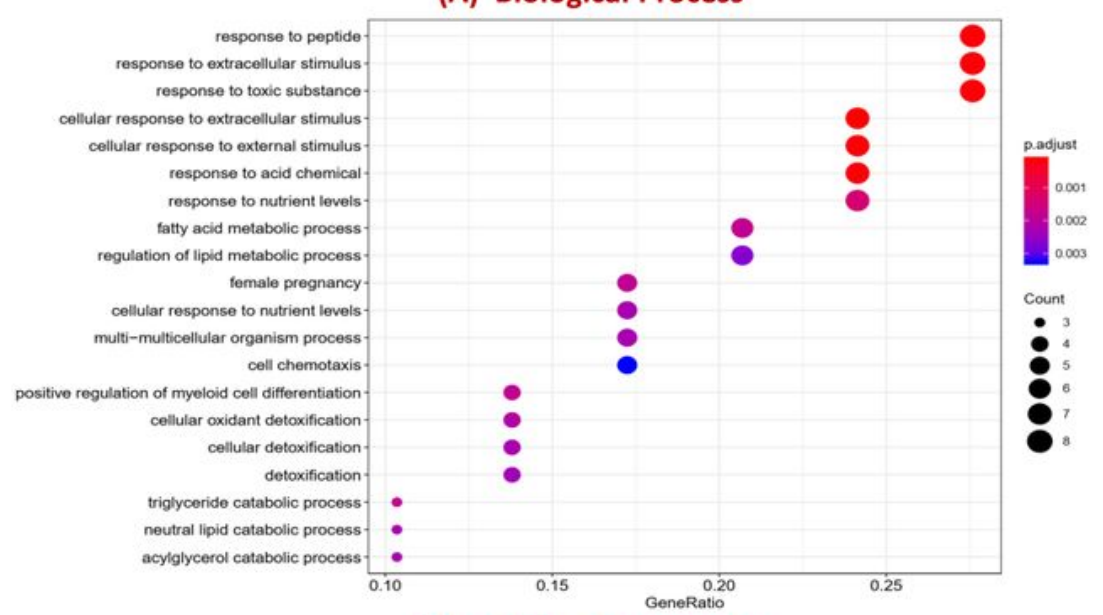

(B) Cellular Components

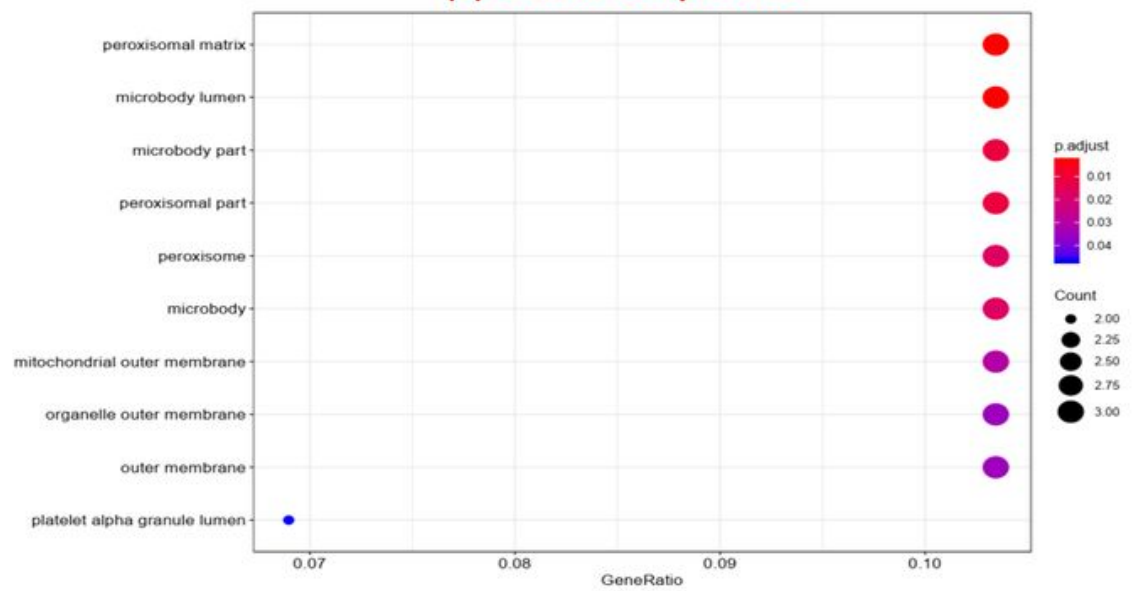

(C) Molecular Function

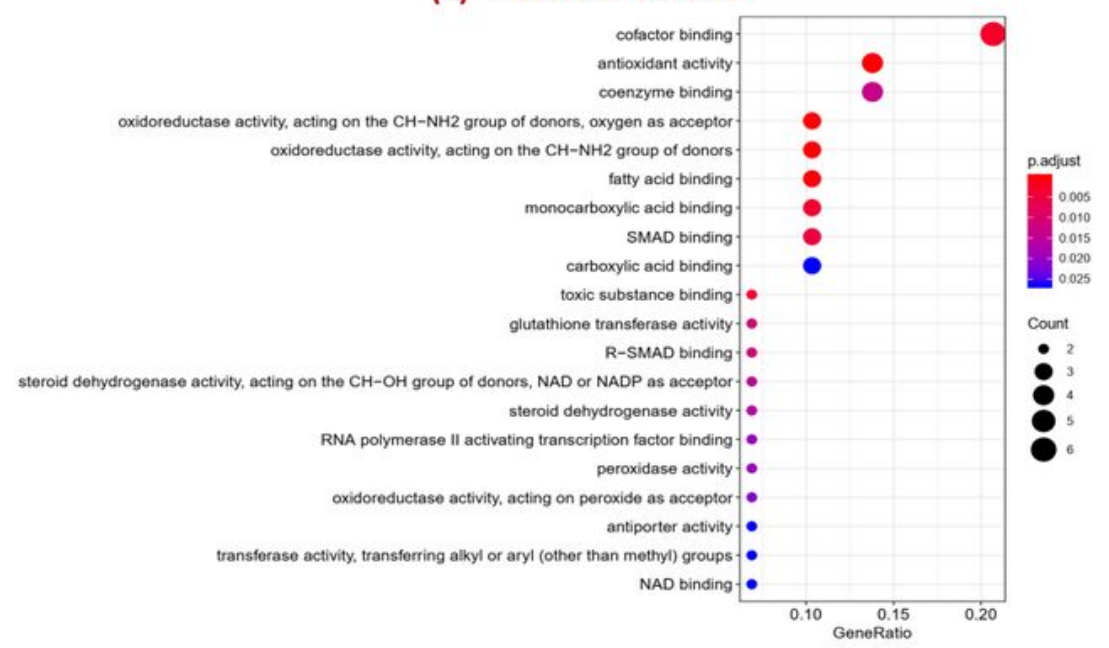

\section{Figure 4}

GO analysis of SED-DKD intersection targets. Note: Only the top 20 terms with the lowest FDR are listed. 


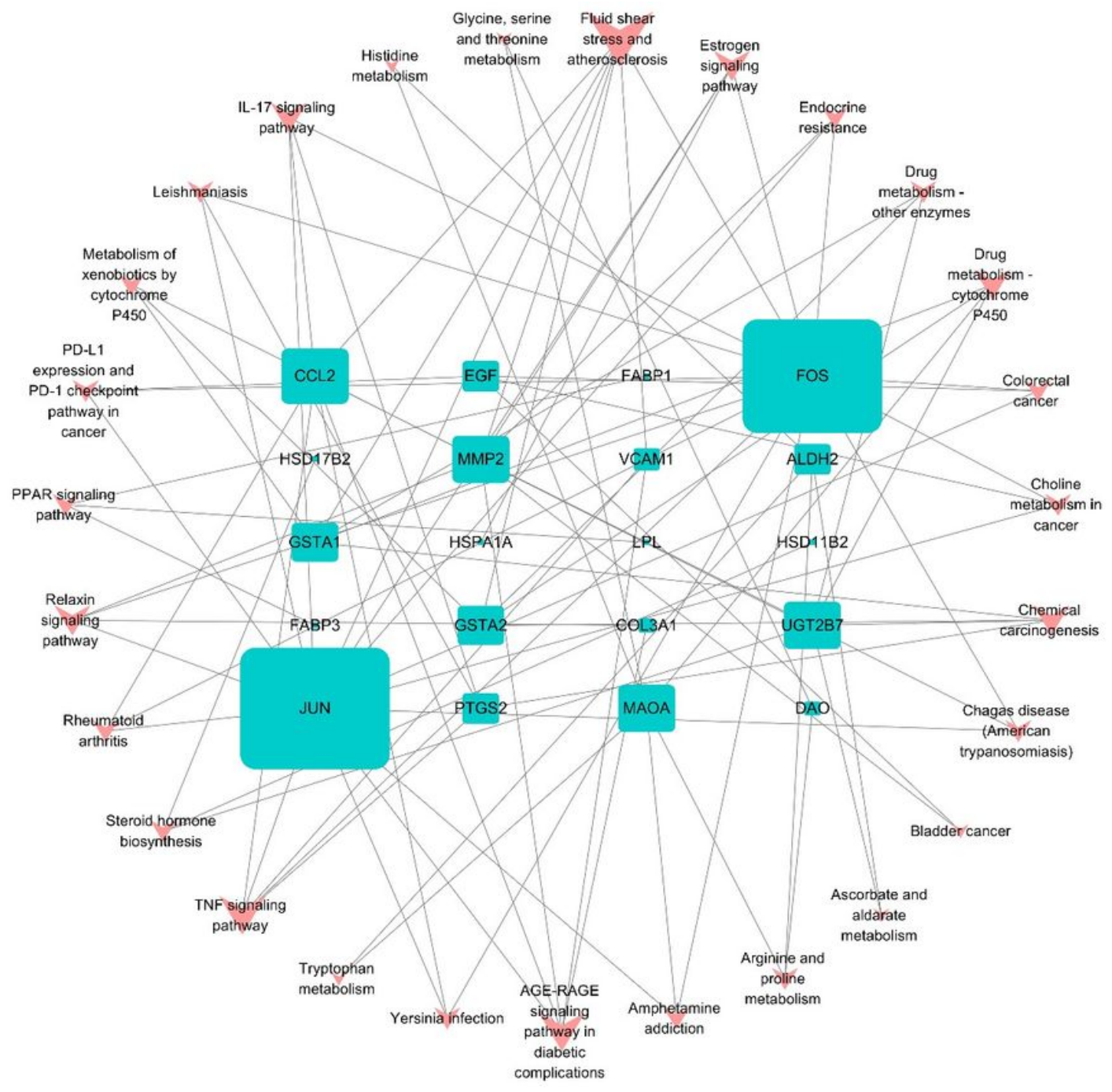

Figure 5

Pathway-target network. 

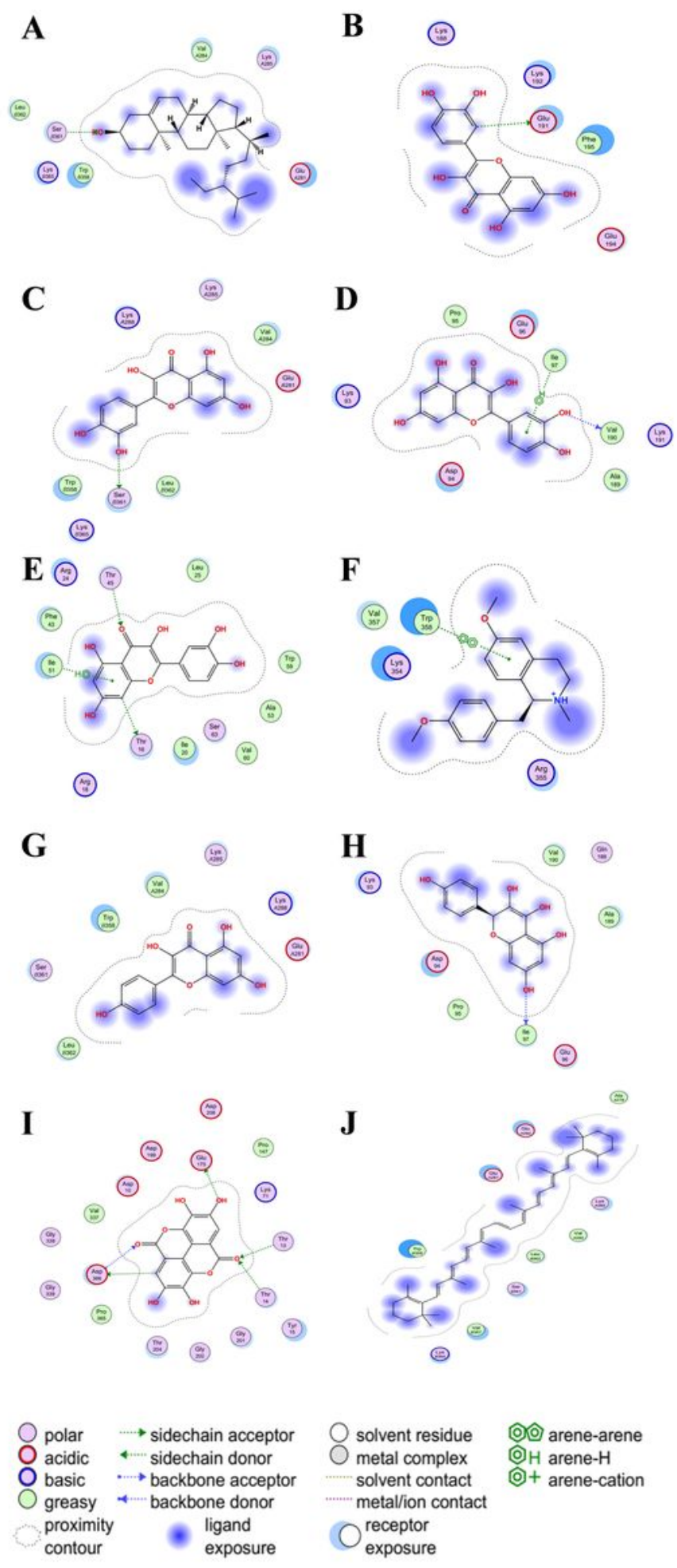

\footnotetext{
$\begin{array}{ll}\text { solvent residue } & \text { 을 arene-arene } \\ \text { metal complex } & \text { 어 } \mathrm{H} \text { arene-H } \\ \text { solvent contact } & \text { 아 }+ \text { arene-cation } \\ \text { metal/ion contact } & \\ \text { receptor } & \\ \text { exposure } & \end{array}$
}

\section{Figure 6}

Mode of molecular docking. Note $₫ A$ stands for the virtual docking of beta-sitosterol and JUN. The virtual docking of quercetin with FOS, JUN, VCAM1, and CCL2 was represented by B, C, D, and E, respectively. F stands for the docking of F4'-Methyl-N-methylcoclaurine and JUN. The docking of kaempferol with JUN and VCAM1 was represented by $\mathrm{G}$ and $\mathrm{H}$, respectively. I stands for the docking of Ellagic acid and HSPAIA. J stands for the docking of beta-carotene and JUN. 


\section{Supplementary Files}

This is a list of supplementary files associated with this preprint. Click to download.

- SupplementaryTable1.xlsx 